\title{
Glenoid Labral Injuries Are More Common Posteriorly Than Superiorly and Are Combined Across Multiple Areas of the Glenoid
}

\author{
W. Ben Kibler, M.D., William J. Grantham, M.D., John Stuart Mattison Pike, B.S., and \\ Aaron D. Sciascia, Ph.D., A.T.C., P.E.S., S.M.T.C., F.N.A.P.
}

\begin{abstract}
Purpose: To categorize arthroscopically observed labral injuries to include location on the glenoid and frequency of the injuries in each location. Methods: Patients undergoing arthroscopic labral surgery between January 2018 and June 2020 were reviewed. Inclusion criteria for labral injury were consistently applied and included history, clinical examination and imaging findings, and failure of rehabilitation. Exclusion criteria included arthritis, adhesive capsulitis, and previous surgery. Injury locations were categorized into superior, anterior, and/or posterior areas on the glenoid and as isolated in one area or combined in more than one area. Injury patterns also were evaluated. Interrater and intrarater agreement was assessed between 2 raters for injury location and tear pattern for 22 randomly assigned cases. Results: In total, 167 cases met the inclusion criteria. Injuries were found in all areas. Combined injuries were found almost twice as often as isolated injuries $(63.5 \%$ vs $37.5 \%)$. Isolated posterior injuries had the greatest frequency of all specific injury types $(26.3 \%)$. Isolated superior injuries (SLAP 2-4) had a frequency of $7.2 \%$. Tear patterns included peel back, separation/split, insubstance injury, peripheral rim flattening, and extension into the posterior inferior glenohumeral ligament. Interrater was excellent for all tear locations (intraclass correlation coefficient $\geq 0.85$ ) whereas intrarater agreement was good to excellent (intraclass correlation coefficient $\geq 0.63$ ). Conclusions: Labral injuries that are associated with clinical symptoms can occur as isolated or combined types in the superior, anterior, and posterior glenoid areas and can display multiple injury patterns. Combined types of injuries are almost twice as common as those that are isolated in one area. Posterior injuries, isolated or combined, are frequent but superior injuries are less common Level of Evidence: Level IV, therapeutic case series.
\end{abstract}

$\mathbf{I}_{\mathrm{a}}^{\mathrm{n}}$ njuries involving the glenoid labrum, both from acute trauma and from chronic microtrauma, frequently are associated with shoulder dysfunction. They are most commonly classified into anterior and posterior injuries, mainly associated with acute

From the Shoulder Center of Kentucky, Lexington Clinic, Lexington, Kentucky (W.B.K., W.J.G.); Medical University of South Carolina, Charleston, South Carolina (J.S.M.P.); and Department of Exercise and Sport Science, Eastern Kentucky University, Richmond, Kentucky (A.D.S.), U.S.A.

The authors report that they have no conflicts of interest in the authorship and publication of this article. Full ICMJE author disclosure forms are available for this article online, as supplementary material.

Address correspondence to Aaron D. Sciascia, Ph.D., A.T.C., P.E.S., S.M.T.C., F.N.A.P., Department of Exercise and Sport Science, Eastern Kentucky University, 228 Moberly Building, 521 Lancaster Ave., Richmond, KY40475.E-mail: aaron.sciascia@eku.edu

(C) 2021 THE AUTHORS. Published by Elsevier Inc. on behalf of the Arthroscopy Association of North America. This is an open access article under the CC BY-NC-ND license (http://creativecommons.org/licenses/by-nc-nd/4.0/). 2666-061X/21981

https://doi.org/10.1016/j.asmr.2021.11.014 traumatic mechanisms, and SLAP injuries associated with microtrauma mechanisms. SLAP injuries ${ }^{1,2}$ have been considered to be major causative factors in repetitive microtrauma based shoulder pain and dysfunction in patients involved in overhead sports and work activities. Multiple studies have examined the mechanisms of SLAP injury production, methods of examination and identification of the injury, and techniques of operative treatment. ${ }^{1-9}$ However, outcomes of treatment of the SLAP lesion vary widely, suggesting incomplete understanding of the exact pathoanatomy of the injury and resultant lack of clarity regarding treatment. ${ }^{4-12}$

Labral injuries were originally classified into 4 types in relationship to the SLAP lesion, ${ }^{2}$ and the original classification has been expanded around the glenoid face to include other areas of injury that have been found to exist, resulting in more subtypes. ${ }^{13}$ The focus of the classifications has been mainly on identifying and treating microtrauma labral injuries in the context of the superior based SLAP injury and labral injuries 
Table 1. Clinical Diagnosis Criteria

\begin{tabular}{lc}
\hline & Patient History \\
\hline $\begin{array}{l}\text { Pain and/or dysfunction during overhead activities (work, sport, and/or activities of } \\
\text { daily living) }\end{array}$ & Positive active compression test \\
Pain and/or dysfunction during throwing with the arm in cocking, abduction with & Positive modified dynamic labral shear (M-DLS) \\
$\quad$ external rotation, and follow-through & Positive Kim's I and II biceps load test \\
A feeling of "difficulty getting loose" & Altered glenohumeral total range of motion \\
A complaint or feeling of instability (apprehension, sliding, catching, or “dead arm") & Positive apprehension/relocation test \\
\hline
\end{tabular}

NOTE. At least 4 of the history and physical examination criteria had to be demonstrated.

associated with acute trauma based glenohumeral instability into mainly anterior or posterior groups., ${ }^{2,14}$ Recent studies, however, demonstrate that injuries in other parts of the labrum, especially the posterior labrum, may be frequent and clinically relevant in both types of labral injuries. ${ }^{16-19}$ Also, a current concepts consensus review suggested that the classical SLAP injury, a detachment from the 11:00 to the 1:00 position on the glenoid, may actually be an adaptive event to allow maximal external rotation in cocking and may not represent clinically significant pathoanatomy. ${ }^{3}$ These findings suggest a need for further investigations confirming these new insights and providing improved scholarship regarding the exact extent of the pathoanatomy of the labral injury in all areas of the glenoid and in all types of injury. Further confirmation of these findings could help improve arthroscopic evaluation techniques for the labral injury associated with clinical symptoms and help to guide comprehensive treatment.

The purpose of this study was to categorize arthroscopically observed labral injuries to include location on the glenoid and frequency of the injuries in each location. The hypotheses of this study were that labral injuries would occur in multiple areas on the glenoid and would have multiple injury patterns, and that posterior labral injuries would occur frequently.

\section{Methods}

Chart and arthroscopic videos and images of all patients who underwent arthroscopic surgery on the shoulder (Current Procedural Terminology codes 29806, 29807, 29822, and 29823) at our shoulder center from January 2018 to June 2020 were reviewed. Cases were contributed from the practices of 4 shoulder specialists within one practice. The senior author reviewed all cases to determine whether the inclusion criteria were consistently applied and were met. Inclusion criteria used the same components developed for a previous study: age 14-55 years; standardized clinical history, clinical examination, and imaging findings indicative of a clinical diagnosis of labral injury; failure to improve their clinical symptoms and dysfunction following a specific rehabilitation program designed to address the musculoskeletal deficits identified on the clinical examination ${ }^{20}$; and arthroscopic surgery that identified an anatomic labral injury and included a labral repair.

The exact methodology for achieving the clinical diagnosis of a labral injury that is associated with clinical dysfunction and symptoms is evolving. This study used an established methodology that has been shown to result in reliable data. In this methodology, patients had to demonstrate at least 4 of the history and physical examination criteria from a specific list (Table 1) to be considered for a clinical diagnosis of a labral injury. ${ }^{21}$ This process is based on a modification of criteria for clustering of clinical testing to establish the diagnosis. ${ }^{22}$ Magnetic resonance imaging was considered to be confirmatory but was not used as the single diagnostic criterion due to the demonstrated lack of efficacy as a single diagnostic entity and its poor correlation with clinical symptoms. ${ }^{19,23,24}$ The efficacy and reliability of this diagnostic process for the identification of the clinical diagnosis of a labral injury was shown in a previous study. ${ }^{20}$

Inclusion for arthroscopy also was based on the patient's failure to respond to a specific rehabilitation program within 6 to 8 weeks. ${ }^{20,25}$ The program was designed to address the demonstrated deficits in flexibility, strength, and motion that were discovered on the clinical examination, because studies have shown that a proportion of patients with labral injuries will clinically improve with rehabilitation..$^{20,26,27}$ Exclusion criteria were age $<14$ or $>55$ years; revision surgery; arthritis; adhesive capsulitis or debridement only.

The labral injuries were organized by patient's activity (sports at any level of skill/nonsports) and patientreported mechanism of causation-acute or nonacute. Acute onset was defined as a one-time traumatic incident or a one-time sudden onset of limiting symptoms. Not all acute injuries were dislocations. Nonacute was defined as a gradual onset of worsening and limiting symptoms. Injury location was determined by review of arthroscopic videos and images obtained at the time of surgery from a standardized posterior arthroscopic portal with the patient in the lateral decubitus position but reviewed at a later date. Patients were included in 


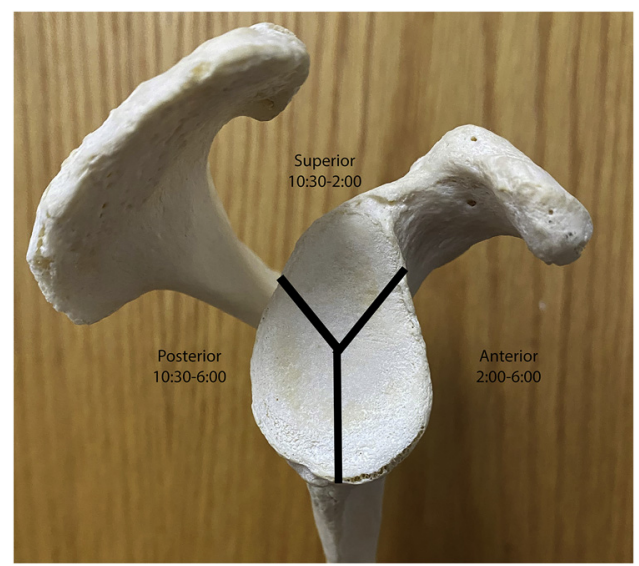

Fig 1. Tear location schema.

the analysis only if the entire glenoid face could be visualized from the available images and video.

The glenoid face was divided into 3 areas (anterior [2:00-6:00 right, 10:00-6:00 left], superior [10:30-2:00 right, 10:00-1:30 left], and posterior [10:30-6:00 right, 1:30-6:00 left]). (Fig 1) Anatomic variants such as a sublabral foramen or Buford complex were differentiated from injuries by the presence of a synovial lining, smooth edges, lack of proliferative synovitis, and/or absence of torn fibers. ${ }^{28}$ The injuries were identified as isolated (injury contained within only one of the defined areas) or combined (injury extending into more than one area). This resulted in injuries being reported as isolated anterior, isolated superior (SLAP 2-4), and isolated posterior, and combined anterior/posterior, combined superior/anterior (SLAP 5), combined superior/posterior (SLAP 8), and combined anterior/superior/posterior (SLAP 9). ${ }^{13}$ The arthroscopically observable injuries were noted to display several different patterns which have been previously categorized. $^{21}$ These included peel back (Fig 2), separation from the glenoid (Fig 3), split with no separation from the glenoid (Fig 4), insubstance injury and/or delamination of the labral contents (Fig 5), flattening of peripheral rim (Fig 6), and/or extension of the injury into the posterior inferior glenohumeral ligament (PIGHL) (Fig 7, Table 2). The injuries were not correlated with patient age. All of the findings were reviewed between July and August 2020 as part of a single reviewing session for each patient case. To prevent bias, no access was provided to the actual operative reports.

Inter- and intrarater reliability was assessed by comparing the observations on the labral location on 22 of the arthroscopic cases, chosen randomly from all cases, between 2 raters (W.B.K. and W.J.G.). The 2 surgeon raters were blinded from all patient documentation and were only allowed to view the captured arthroscopic videos and images from surgery. Patient demographic information was revealed and recorded only after all images were reviewed by both raters and statistical procedures for determining agreement occurred. Using a 2-way random with absolute agreement design for inter-rater $(2, \mathrm{k})$ and intrarater $(2,1)$ test/retest reliability, intraclass correlation coefficients (ICC) were calculated. An ICC $\geq 0.75$ was interpreted as excellent, 0.74-0.60 was good, 0.59-0.40 was fair, and $<0.40$ was considered poor. ${ }^{29}$

\section{Results}

A total of 478 arthroscopic cases were reviewed; of these, 167 (age $=24.7 \pm 8.2$ years; male $=127$, female $=40)$ met the inclusion criteria. $73 \%(122 / 167)$ of the patients had a history of sports activity at some skill level. Twenty-two participated in college sports, 42 in high school sports, and 58 participated in recreational sports. The specific sports and number of participants included baseball/softball (72), football (36), volleyball (6), soccer (3), wrestling (3), and basketball (2). 66\% $(110 / 167)$ had a nonacute mechanism of injury.

\section{Injury Location}

Frequency of injury location by mechanism and activity level is shown in Table 3.

\section{Overall Injuries}

Isolated posterior injuries had the highest frequency of all tear injury types (26.3\% of all injuries), followed by combined anterior/posterior injuries $(24.5 \%)$, combined superior/posterior injuries (SLAP 8) $(22.2 \%)$, and combined superior/anterior/posterior injuries (SLAP 9) $(13.8 \%)$. The frequency of isolated superior injuries (SLAP $2-4)$ was $(7.2 \%)$, isolated anterior $(2.9 \%)$, and combined superior/anterior (SLAP 5) (2.9\%). Some type of injury involving the superior labrum was identified in 77 of 167 cases $(46.1 \%)$, the anterior labrum in 74 of 167 cases $(44.3 \%)$, and the posterior labrum in 145 of 167 cases $(86.8 \%)$.

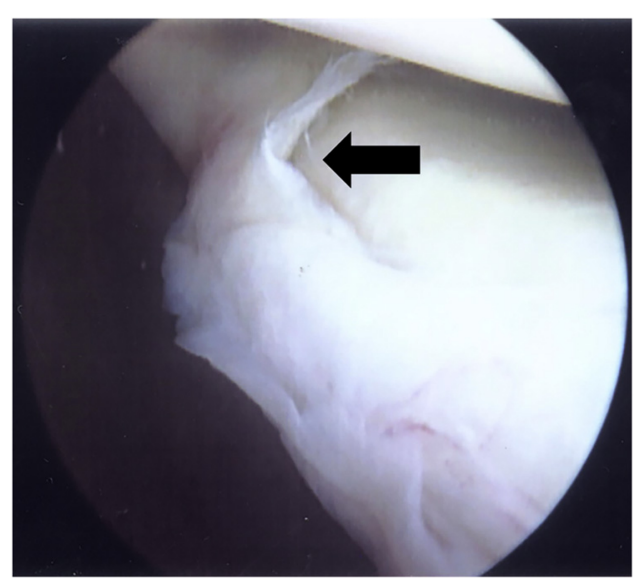

Fig 2. Posterior viewing portal of right shoulder, lateral decubitus position: peel back example. 


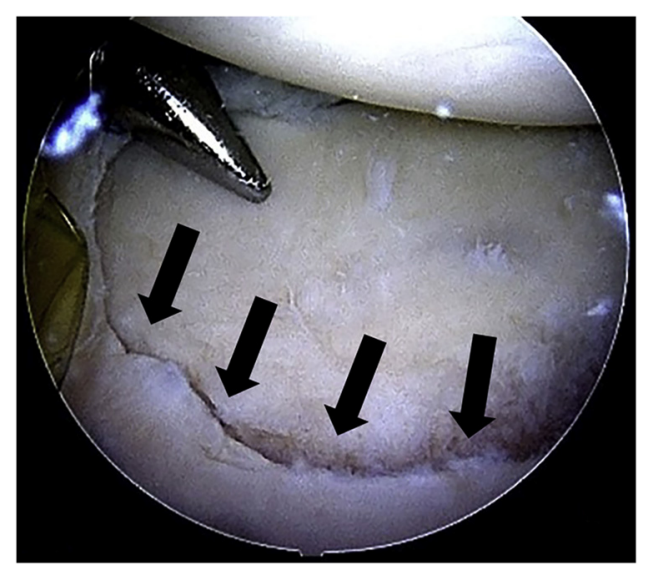

Fig 3. Posterior viewing portal of right shoulder, lateral decubitus position: tear separated from bone example.

\section{Combined Versus Isolated Injuries}

Combined injuries $(n=106)$ were almost 2 times more likely to be identified than isolated injuries $(\mathrm{n}=$ $61)(63.5 \%$ of all injuries vs $36.5 \%$ respectively). Of all isolated injuries $(\mathrm{n}=61)$, the highest frequency of isolated injuries occurred posteriorly ( $\mathrm{n}=44,72.1 \%$ ), followed by isolated superior injuries (SLAP 2-4) $(\mathrm{n}=$ $12,19.7 \%)$ and isolated anterior injuries $(\mathrm{n}=5,8.2 \%)$.

Of all combined injuries $(\mathrm{n}=106)$, anterior/posterior combination tears were the most frequent $(n=41$, $38.7 \%$ ) of all combined injuries followed by superior/ posterior injuries (SLAP 8) $(\mathrm{n}=37,34.9 \%)$, superior/ anterior/posterior injuries (SLAP 9) $(\mathrm{n}=23,21.7 \%)$, and superior/anterior injuries (SLAP 5) $(\mathrm{n}=5,4.7 \%)$.

\section{Mechanisms of Injury}

\section{Injury Types by Acute/Nonacute Mechanism}

Of the nonacute mechanisms $(n=110)$, isolated superior (SLAP 2-4) injuries $(n=12)$, superior/anterior (SLAP 5) injuries $(\mathrm{n}=5$ ), and superior/posterior (SLAP $8)$ injuries $(n=37)$ exclusively occurred in this category (Table 3 ). There were also $35.5 \%$ isolated posterior injuries $(n=39)$ that occurred nonacutely for all injury types. Of the acute onset mechanisms $(n=57)$, there were $57.9 \%$ combined anterior/posterior injuries $(\mathrm{n}=41)$, followed by $26.3 \%$ superior/anterior/posterior (SLAP 9) injuries $(\mathrm{n}=15), 8.8 \%$ isolated posterior injuries $(\mathrm{n}=5)$, and $7.0 \%$ isolated anterior injuries $(\mathrm{n}=4)$. When comparing acute to nonacute mechanisms (Table 4), the majority of the isolated anterior injuries $(\mathrm{n}=4,80 \%)$, combined anterior/posterior injuries $(\mathrm{n}=33,80.5 \%)$, and superior/anterior/posterior injuries ( $\mathrm{n}=15,65.2 \%$ ) occurred acutely, while the majority of isolated posterior injuries $(\mathrm{n}=39,88.6 \%)$ occurred nonacutely.

\section{Injury Types by Sports/Nonsports Activity}

All types of injuries occurred in both sports $(\mathrm{n}=122)$ and nonsports activities $(\mathrm{n}=45)$ (Table 4$)$. When we compared sports with nonsports activity, the large majority of combined anterior/posterior injuries ( $\mathrm{n}=$ $36 / 41,87.8 \%$ of all anterior/posterior injuries), combined superior/anterior/posterior injuries (SLAP 9) $(\mathrm{n}=18 / 23,78.3 \%)$, isolated posterior injuries $(\mathrm{n}=33$ / $44,75 \%)$, and combined superior/posterior injuries (SLAP 8$)(\mathrm{n}=26 / 37,70.3 \%)$ occurred in sports activities. But up to $30 \%$ of these injuries occurred in nonsports activities. Isolated superior injuries (SLAP 2-4) were more evenly distributed between sports $(n=5 /$ $12,41.7 \%)$ and nonsports $(\mathrm{n}=7 / 12,58.3 \%)$ activities.

\section{Injury Patterns}

Most injuries were noted to be separations from the glenoid $(\mathrm{n}=109,65.2 \%)$. The second most identified pattern was insubstance injury with delamination which occurred in $52.7 \%$ of all injuries $(\mathrm{n}=88)$. Peelback $(\mathrm{n}=79)$ and a split where the bone could not be visualized $(\mathrm{n}=76)$ occurred similarly, $47.3 \%$ and $45.5 \%$ respectively. Flattening of the peripheral rim $(\mathrm{n}=65,38.9 \%)$ and injury extending into the PIGHL $(\mathrm{n}=56,33.5 \%)$ was present in just more than onethird of all injuries. More than one pattern could be identified in many cases (Fig $8 \mathrm{~A}$ and B).

\section{Inter- and Intrarater Agreement}

The agreement results are shown in Table 5. When we examined the results for injury location and frequency, inter-rater reliability was excellent for all injury locations (ICC $\geq 0.83$ ). Intrarater reliability for examiner 1 was rated as excellent for all injury locations (ICC $\geq 0.93$ ), whereas intrarater for examiner 2 ranged from good to excellent (ICC $\geq 0.63$ ).

\section{Discussion}

The data provide evidence to accept the hypotheses. Patients were found on arthroscopy to have labral

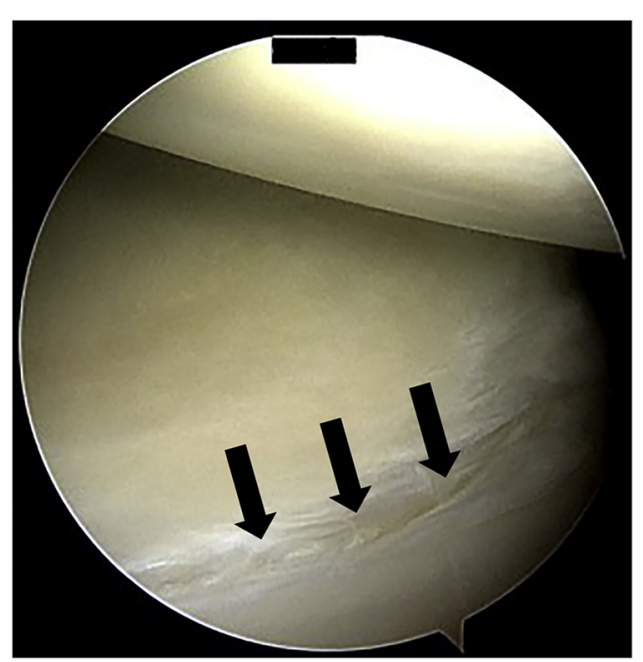

Fig 4. Posterior viewing portal of right shoulder, lateral decubitus position: split example. 


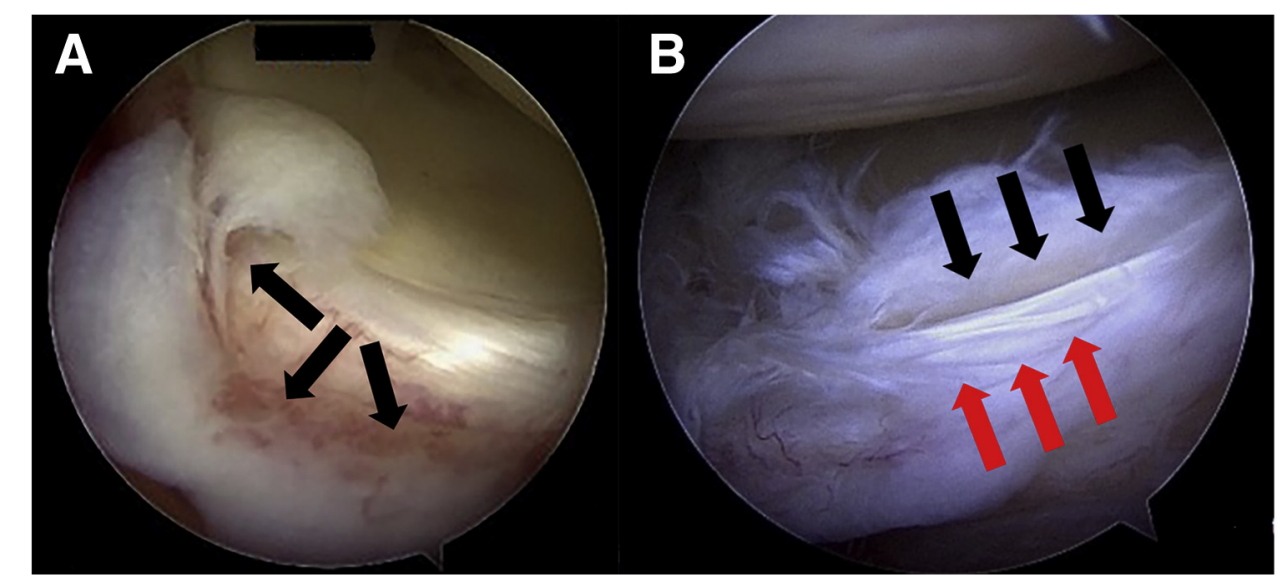

Fig 5. (A) Posterior viewing portal of right shoulder, lateral decubitus position: insubstance shear and delamination example. (B) Posterior viewing portal of right shoulder, lateral decubitus position: split (black arrows) and insubstance delamination (red arrows) example.

injuries at varying locations around the superior, anterior, and posterior glenoid face. Posterior labral injuries, isolated or in combination with other injuries, were common. In addition, combined injuries occurring in more than area were almost twice as common as isolated injuries.

The superior labrum detachment from the glenoid (SLAP injury) has been thought to be foundational to the dysfunction seen in the disabled throwing shoulder ${ }^{3}$ and injury in workers. ${ }^{2}$ Treatment outcomes using these concepts and focusing on treatment of the superior-based injury have not been consistent, with variable return to activity and a high number of "failed" SLAP repairs, ${ }^{10,30}$ suggesting that other types of injury and pathoanatomy may be additional factors in the dysfunction. In addition, these findings indicate that labral injuries associated with glenohumeral instability may have more extensive tissue damage around the glenoid. Taken together, these findings are consistent with anecdotal clinical experience from several centers and from current papers, $3,16-19,31,32$ which suggest that labral injuries that are associated with clinical symptoms may have variable locations of pathology. In addition, the observed injuries may present variable types of injury patterns each of may alter the labral roles that are considered keys in helping confer dynamic concavity/compression for the glenohumeral joint. ${ }^{33,34}$ Labral injuries also were found with more frequency in combined locations than in isolated locations. These findings confirm other studies and suggest revisiting of the concept of the pathoanatomy of the labral injury associated with clinical symptoms.

\section{Location and Frequency of Injury}

Most previous studies describing treatment of SLAP or instability injuries have not described the exact location of the injuries on the glenoid. Some have described variations of the SLAP injury extending to other locations on the glenoid but did not report the exact criteria used to determine the location or the frequency of the injuries in each location. ${ }^{13-15,35,36}$ A recent paper did evaluate specific location of labral injuries around the glenoid and used 3 anatomic areas and 10 categories of injury. ${ }^{19}$ For this study, the glenoid was divided into 3 areas, based on previous descriptions of classification of labral injuries. Specific criteria were developed to define the areas and categorize the location of the injuries, and all areas of the glenoid face were specifically evaluated to categorize the frequency of each injury in each area. The superior section was defined from 10:30-2:00 on the right shoulder, and 10:00-1:30 on the left shoulder. This anatomic area is more rounded and mobile, has been categorized as a mobile organ of tension, a functional tension band, ${ }^{28,37}$ and encompasses the anatomic area found to be the anchor point for optimum distribution of tensile loads on the biceps labrum complex. ${ }^{31}$ The anterior and posterior sections were defined running from their superior demarcation down to the 6:00 position and encompassing the inferior labrum.

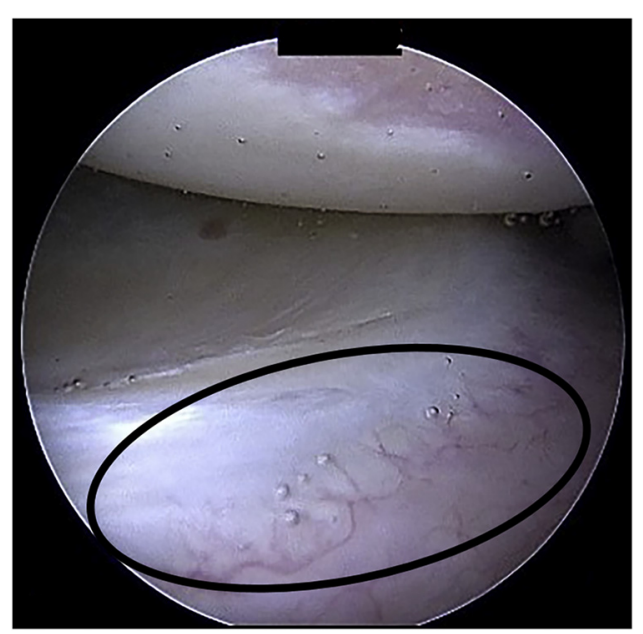

Fig 6. Posterior viewing portal of right shoulder, lateral decubitus position: flattening of the posterior labrum example. 


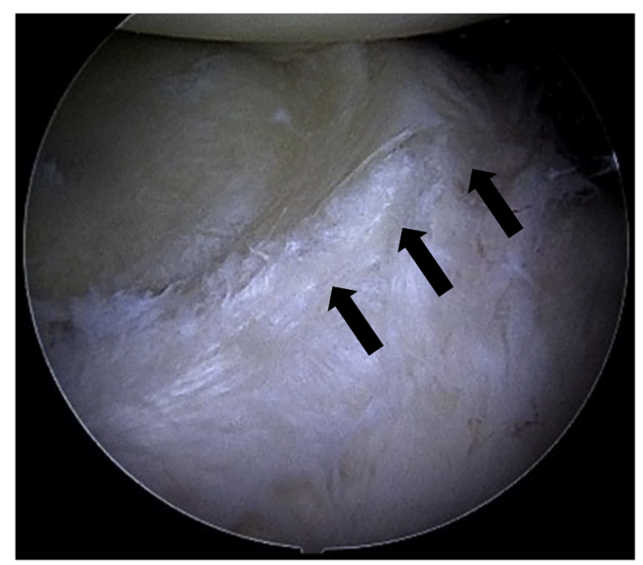

Fig 7. Posterior viewing portal of right shoulder, lateral decubitus position: extension of injury into the posterior inferior glenohumeral ligament example.

The inferior labrum is well attached and immobile, and has been categorized as a fixed organ of compression, a bumper conferring edge stability against translation. ${ }^{28,37}$ This method of division also conforms to most previous descriptions and appears to generate good reliability for analysis, based on the interrater agreement values. The one category with only moderate agreement was the posterior injury. Review of these cases showed that the major difference in agreement was not about determining the presence of the posterior injury but occurred in deciding whether to call the injury an isolated posterior injury or a SLAP 8 injury, with a slight difference in determining the extent of superior involvement.

One of the major findings of this study was that the frequency of combined injuries was almost twice that of isolated injuries ( $63.5 \%$ vs $36.5 \%)$, and that both acute and nonacute mechanisms of injury demonstrated this finding. This may reflect the evaluation criteria that any part of the injury that could be observed to extend into more than one anatomic area be included as a combined injury. However, while this method of evaluation may not identify the most extensive part of the injury (a Bankart lesion extending from 3:00 around to 7:00 on the right shoulder or a posterior labral injury extending from 10:00 down to 6:30 on the right shoulder), this finding does highlight the need to be comprehensive in the arthroscopic evaluation of all areas on the glenoid, is consistent with the findings that superior injuries under tension tend to propagate posteriorly ${ }^{38}$ and anterior injuries under load to propagate posteriorly, and is consistent with the patterns demonstrated in a recent study evaluating labral injury locations. ${ }^{19}$ This study did not use as many categories of labral injury locations but did agree that many labral tears are extensive in location and can only be described as combination injuries. The low incidence of isolated anterior $(7.3 \%)$ and posterior $(9.1 \%)$ labral injuries in acute injuries suggests that clinically symptomatic instabilities are infrequently associated with localized isolated labral injury but are more likely to propagate around the 6:00 position, ${ }^{39}$ data that confirm recent findings. ${ }^{19}$ This would be in agreement with the "Circle concept" of glenohumeral instability. ${ }^{40}$

Another major finding was that posterior labral injuries, either a separation or split from the glenoid or an insubstance delamination, were common, either in combination with injury in other locations or as an isolated injury. There was a high incidence of isolated posterior injuries $(26.9 \%$ of all injuries, $34.8 \%$ of nontraumatic acute injuries), and SLAP 8 injuries (22.1\% of all injuries, $33 \%$ of nonacute injuries), and some type of labral injury involving the posterior labrum was observed in $86.8 \%$ of all cases, confirming previous studies that suggested a high frequency of this injury and emphasizing the need to evaluate and treat

Table 2. Injury Patterns

\begin{tabular}{|c|c|}
\hline Pattern & Description \\
\hline Peel back & $\begin{array}{l}\text { Observable motion of the superior and posterior labrum so that it } \\
\text { rolled off the glenoid face as the arm was taken from internal } \\
\text { rotation to external rotation }\end{array}$ \\
\hline Tear & $\begin{array}{l}\text { Visible separation of the labrum from the glenoid, with complete } \\
\text { separation from the glenoid so that the underlying bone could be } \\
\text { visualized, directly upon visualization or in association with the pee } \\
\text { back }\end{array}$ \\
\hline Split & $\begin{array}{l}\text { Visual separation of the labrum from the glenoid, with a crack in the } \\
\text { articular side surface of the labral attachment, with no bone } \\
\text { visualized }\end{array}$ \\
\hline Insubstance shear or delamination & $\begin{array}{l}\text { Damage (tear or a flap of tissue) to the superficial layer of the labrum, } \\
\text { with visualization of the intermediate layer and its striated bundles, } \\
\text { with or without a tear away from the bone }\end{array}$ \\
\hline Flattening of the posterior labrum & $\begin{array}{l}\text { Observation of the labral surface flattened away from its position on } \\
\text { the glenoid, so it is not a bumper }\end{array}$ \\
\hline Extension into the posterior inferior glenohumeral ligament (PIGHL) & $\begin{array}{l}\text { Extension of the tear into the attachment of the PIGHL, with or } \\
\text { without extension continuing into the inferior labrum }(6: 00)\end{array}$ \\
\hline
\end{tabular}


Table 3. Frequency of Injury Location by Mechanism and Activity Level (Presented as Frequency [Percentage])

\begin{tabular}{|c|c|c|c|c|c|}
\hline \multirow[b]{2}{*}{ Location } & \multirow[b]{2}{*}{$\begin{array}{c}\text { Overall } \\
\mathrm{n}=167\end{array}$} & \multicolumn{2}{|c|}{ Mechanism } & \multicolumn{2}{|c|}{ Activity Level } \\
\hline & & $\begin{array}{c}\text { Acute } \\
\mathrm{n}=57\end{array}$ & $\begin{array}{c}\text { Nonacute } \\
\mathrm{n}=110\end{array}$ & $\begin{array}{c}\text { Sport } \\
\mathrm{n}=122 \\
\end{array}$ & $\begin{array}{c}\text { Nonsport } \\
\mathrm{n}=45\end{array}$ \\
\hline Isolated superior(SLAP 2-4) & $12(7.2 \%)$ & $0(0.0 \%)$ & $12(10.9 \%)$ & $5(4.1 \%)$ & $7(15.6 \%)$ \\
\hline Isolated anterior & $5(2.9 \%)$ & $4(7.0 \%)$ & $1(0.9 \%)$ & $3(2.5 \%)$ & $2(4.4 \%)$ \\
\hline Isolated posterior & $44(26.3 \%)$ & $5(8.8 \%)$ & $39(35.5 \%)$ & $33(27.0 \%)$ & $11(24.4 \%)$ \\
\hline Total isolated & $61(36.5 \%)$ & $9(15.8 \%)$ & $52(47.3 \%)$ & $41(33.6 \%)$ & $20(44.4 \%)$ \\
\hline Anterior/posterior & $41(24.5 \%)$ & $33(57.9 \%)$ & $8(7.1 \%)$ & $36(29.5 \%)$ & $5(11.1 \%)$ \\
\hline Superior/anterior (SLAP 5) & $5(2.9 \%)$ & $0(0.0 \%)$ & $5(4.5 \%)$ & $1(0.8 \%)$ & $4(8.9 \%)$ \\
\hline Superior/posterior (SLAP 8) & $37(22.2 \%)$ & $0(0.0 \%)$ & $37(33.6 \%)$ & $26(21.3 \%)$ & $11(24.4 \%)$ \\
\hline Superior/anterior/posterior (SLAP 9) & $23(13.8 \%)$ & $15(26.3 \%)$ & $8(7.1 \%)$ & $18(14.8 \%)$ & $5(11.1 \%)$ \\
\hline Total combined & $106(63.5 \%)$ & $48(84.2 \%)$ & $58(52.7 \%)$ & $81(66.4 \%)$ & $25(55.6 \%)$ \\
\hline
\end{tabular}

this injury in patients with labral injuries associated with clinical symptoms. ${ }^{10,19,30}$

A third finding of this study was the relatively low frequency of injuries involving the superior labrum. The frequency of isolated superior injuries, SLAP 2-4, was low, especially in sports activities $(4.1 \%$ of all sports injuries, $41.6 \%$ of all isolated superior injuries). This low frequency of total injuries and sports-related injuries is consistent with the data from Snyder's original description of the superior injury. ${ }^{2}$ The finding that some type of injury involving the superior labrum was identified in only $46.1 \%$ cases, while injury involving the anterior labrum was identified in $44.3 \%$ cases, and the posterior labrum in $86.8 \%$ cases emphasizes the need to evaluate the entire labrum before making the arthroscopic diagnosis of an isolated superior or anterior labral injury. Considering that superior/posterior injuries (SLAP 8), isolated superior injuries (SLAP 2-4), and superior/ anterior injuries (SLAP 5) occurred exclusively through non acute mechanisms, and that all the other injury types occurred in both mechanisms, the need to evaluate the entire labrum in all cases of acute injury and many of the nonacute cases, to discover the entire pathoanatomy is necessary. Similarly, all types of injuries occurred in both sports and nonsports activities requiring a comprehensive evaluation in all those cases.

\section{Injury Patterns}

As injury location was being evaluated, multiple patterns of anatomic injury were observed. In previous studies, various descriptions of the injury patterns have been listed, including some papers that did not describe criteria for the labral injury. The most common descriptions included a peel back, ${ }^{32,41}$ a separation from the superior glenoid, ${ }^{1,2}$ injury to the biceps attachment, ${ }^{11}$ or a Bankart lesion. ${ }^{40}$ This study used criteria developed for a previous survey of treatment options to organize the appearance of injuries, ${ }^{21}$ with no attempt to provide a new classification system. By their appearance, these injuries could disrupt the normal 3 layer anatomy of the labrum ${ }^{42}$ and could affect the multiple well delineated roles of the labrum as a tension band (injuries involving the superior component with a peel back), a bumper to resist translation and provide edge stability (separation or a split), a washer to distribute loads (insubstance delamination), a deepener of the glenoid socket (flattening of the labrum), or as an attachment site for the glenohumeral ligaments (extension of the tear/split into the PIGHL), which have the common purpose of optimizing dynamic concavity/compression, $32-34,43-45$ so any anatomic finding displaying these characteristics was considered an injury.

Table 4. Between Group Frequency by Mechanism and Activity Level (Presented as Frequency (Percentage)

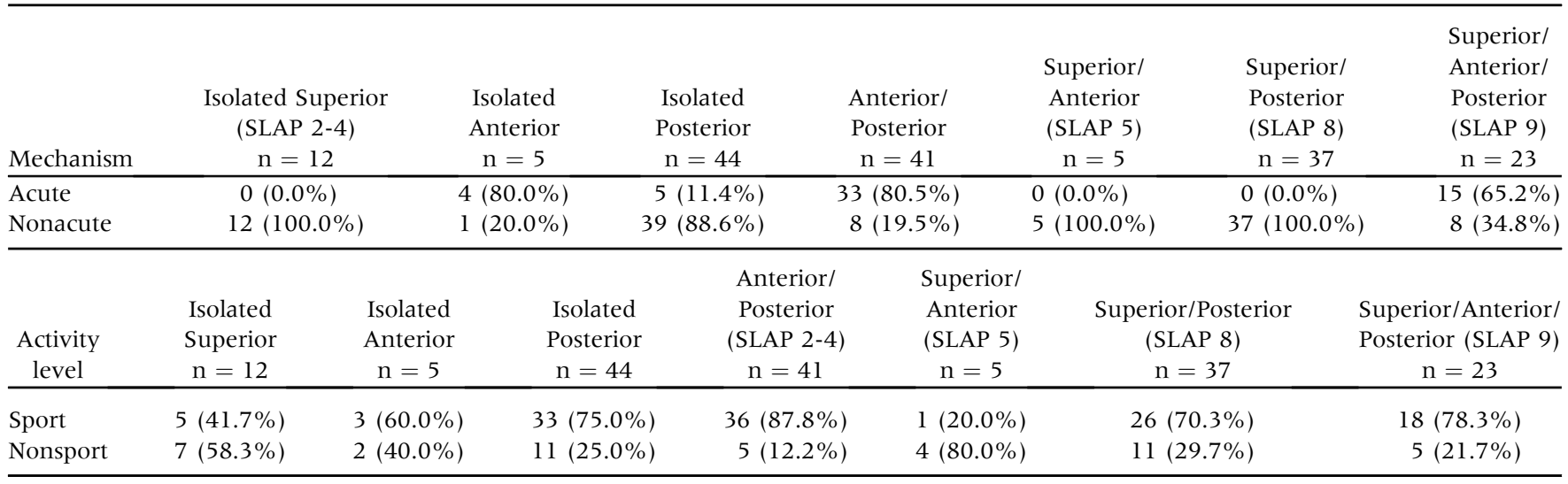




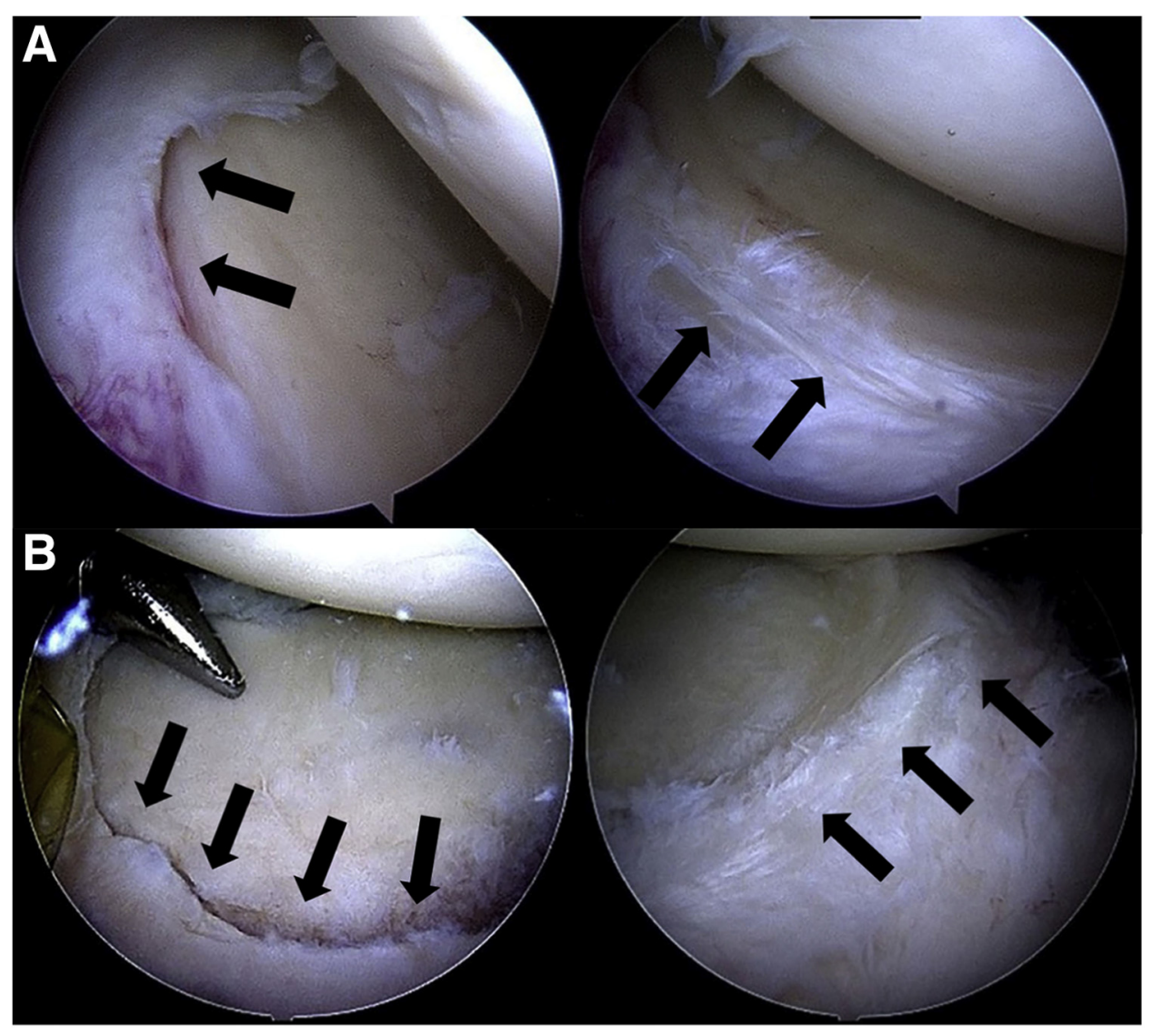

Fig 8. (A) Posterior viewing portal of right shoulder, lateral decubitus position: combined tear and insubstance delamination example. (B) Posterior viewing portal of right shoulder, lateral decubitus position: combined tear and extension into posterior inferior glenohumeral ligament example.

\section{Limitations}

There are several limitations in this study. First, it is a retrospective study of existing data, relying on images and videos, with no "real-time" dynamic analysis, which may result in being unable to identify certain locations of injury. Second, it is only a descriptive analysis of the arthroscopic findings at the time of the surgical procedure in a group of patients who demonstrated the clinical findings of a labral injury based on a specific set of inclusion criteria, so it may not encompass the entire possible clinical presentation of all patients with a labral injury. The intent of this study was to identify the location of the labral injuries, not to determine whether surgery was indicated. This descriptive analysis of arthroscopic findings did not investigate the specific clinical examination findings relating to each patient or type of labral injury, try to identify any correlation of imaging to the clinical diagnosis or arthroscopic findings, examine the surgical techniques of the repair, or report the clinical outcomes. These elements should be evaluated as part of a comprehensive single or multicenter clinical trial. Finally, external validity of these data may be impacted since they are representative of a specific shoulder practice, with a high volume of overhead athletes and workers, and may not be representative of practices that may treat more patients with acute onset injuries or a more general mix of patients.

Table 5. Agreement Between Raters for Injury Location and Injury Patterns $(n=22)$

\begin{tabular}{lccc}
\hline \multicolumn{1}{c}{ Injury Location } & Inter-rater $(95 \% \mathrm{CI})$ & Examiner 1 Intrarater $(95 \% \mathrm{CI})$ \\
\hline Isolated superior & $1.00(\mathrm{~N} / \mathrm{A})$ & $1.00(\mathrm{~N} / \mathrm{A})$ & $\mathrm{Examiner} 2$ Intrarater $(95 \% \mathrm{CI})$ \\
Isolated anterior & $1.00(\mathrm{~N} / \mathrm{A})$ & $1.00(\mathrm{~N} / \mathrm{A})$ & $1.00(\mathrm{~N} / \mathrm{A})$ \\
Isolated posterior & $0.85(0.70,0.93)$ & $0.93(0.83,0.97)$ \\
Anterior/posterior & $0.90(0.81,0.95)$ & $1.00(\mathrm{~N} / \mathrm{A})$ & $0.70(0.26,0.87)$ \\
Superior/anterior & $1.00(\mathrm{~N} / \mathrm{A})$ & $0.96(0.89,0.98)$ & $0.63(0.10,0.85)$ \\
Superior/posterior & $0.93(0.87,0.97)$ & $1.00(\mathrm{~N} / \mathrm{A})$ & $0.90(0.77,0.96)$ \\
Superior/anterior/posterior & $1.00(\mathrm{~N} / \mathrm{A})$ & $1.00(\mathrm{~N} / \mathrm{A})$ & \\
\hline
\end{tabular}

95\% CI, 95\% confidence interval; N/A, not applicable; PIGHL, posterior inferior glenohumeral ligament. 


\section{Conclusions}

Labral injuries that are associated with clinical symptoms can occur as isolated or combined types in the superior, anterior, and posterior glenoid areas and can display multiple injury patterns. Combined types of injuries are almost twice as common than as those that are isolated in one area. Posterior injuries, isolated or combined, are frequent but superior injuries are less common.

\section{Acknowledgments}

The authors thank David Dome, M.D., and Peter Hester, M.D., for their involvement and contribution of their cases for the study.

\section{References}

1. Andrews JR. Glenoid labrum tears related to the long head of the biceps. Am J Sports Med 1985;13:337-341.

2. Snyder SJ, Karzel RP, Del Pizzo W, Ferkel RD, Friedman MJ. SLAP lesions of the shoulder. Arthroscopy 1990;6:274-279.

3. Kibler WB, Kuhn JE, Wilk KE, et al. The disabled throwing shoulder-spectrum of pathology: 10 year update. Arthroscopy 2013;29:141-161.

4. Brockmeier SF, Voos JE, Williams RJ III, Altchek DW, Cordasco FA, Allen AA. Outcomes after arthroscopic repair of type-II SLAP lesions. J Bone Joint Surg Am 2009;91:1595-1603.

5. Radkowski CA, Chhabra A, Baker CL Iii, Tejwant SG, Bradley JP. Arthroscopic capsulolabral repair for posterior shoulder instability in throwing athletes compared with nonthrowing athletes. Am J Sports Med 2008;36:693-699.

6. Katz LM, Hsu S, Miller SL, et al. Poor outcomes after SLAP repair: Descriptive analysis and prognosis. Arthroscopy 2009;25:849-855.

7. Gorantla K, Gill C, Wright RW. The outcome of type II SLAP repair: A systematic review. Arthroscopy 2010;26:537-545.

8. Denard PJ, Ladermann A, Burkhart SS. Long-term outcome after arthroscopic repair of type II SLAP lesions: Results according to age and workers' compensation status. Arthroscopy 2012;28:451-457.

9. Smith R, Lombardo DJ, Petersen-Fitts GR, et al. Return to play and prior performance in major league baseball pitchers after repair of superior labral anterior-posterior tears. Orthop J Sports Med 2016;28:2325967116675822.

10. Sayde WM, Cohen SB, Ciccotti MG, Dodson CC. Return to play after type II superior labral anterior-posterior lesion repairs in athletes. Clin Orthop Rel Res 2012;470: 1595-1600.

11. Provencher MT, McCormick F, Dewing C, McIntire S, Solomon D. A prospective analysis of 179 type 2 superior labrum anterior and posterior repairs: Outcomes and factors associated with success and failure. Am J Sports Med 2013;41:880-886.

12. Kibler WB, Sciascia A. Current practice for the treatment of superior labral anterior to posterior (SLAP) lesions: A systematic review. Arthroscopy 2016;32:669-683.

13. Powell SE, Nord KD, Ryu RKN. The diagnosis, classification, and treatment of SLAP lesions. Op Tech Sports Med 2004:20:99-110.
14. Kim TK, Queale WS, Cosgarea AJ, McFarland EG. Clinical features of the different types of SLAP lesions: An analysis of one hundred and thirty-nine cases. J Bone Joint Surg Am 2003:85:66-71.

15. Kim SH, Ha KI, Yoo JC, Noh KC. Kim's lesions: An incomplete and concealed avulsion of the posteroinferior labrum. Arthroscopy 2004;20:712-720.

16. Sheean AJ, Kibler WB, Conway J, Bradley JP. Posterior labral injury and glenohumeral instability in overhead athletes: Current concepts for diagnosis and management. J Am Acad Orthop Surg 2020;28:628-637.

17. Sheean AJ, Arner JW, Bradley JP. Posterior glenohumeral instability: Diagnosis and management. Arthroscopy 2020;36: 2580-2582.

18. Mannava S, Frangiamore SJ, Murphy CP, et al. Prevalence of shoulder labral injury in collegiate football players at the National Football League scouting combine. Orthop J Sports Med 2018;6:2325967118783982.

19. Alexeev M, Kercher JS, Levina Y, Duralde XA. Variability of glenoid labral tear patterns: A study of 280 sequential surgical cases. J Shoulder Elbow Surg 2021;30:2762-2766.

20. Moore-Reed SD, Kibler WB, Sciascia AD, Uhl T. Preliminary development of a clinical prediction rule for treatment of patients with suspected SLAP tears. Arthroscopy 2014;30:1540-1549.

21. Kibler WB, Sciascia A. Current practice for the diagnosis of a superior labral anterior to posterior (SLAP) lesion: Systematic review and physician survey. Arthroscopy 2015;31:2456-2469.

22. Walsworth MK, Doukas WC, Murphy KP, Mielcarek BJ, Michener LA. Reliability and diagnostic accuracy of history and physical examination for diagnosing glenoid labral tears. Am J Sports Med 2008;36:162-168.

23. Saqib R, Harris J, Funk L. Comparison of magnetic resonance arthrography with arthroscopy for imaging of shoulder injuries: Retrospective study. Ann R Coll Surg Engl 2017;99: 271-274.

24. Connor PM, Banks DM, Tyson AB, Coumas JS, D'Alessandro DF. Magnetic resonance imaging of the asymptomatic shoulder of overhead athletes: A 5-year follow-up study. Am J Sports Med 2003;31:724-227.

25. Moore-Reed SD, Seekins KA, Kibler WB, Sciascia AD, Uhl TL. Conservative treatment for patients with suspected SLAP tears: A case series. Turkiye Klinikleri J Health Sci 2017;2:121-128.

26. Edwards SL, Lee JA, Bell JE, et al. Nonoperative treatment of superior labrum anterior posterior tears: Improvements in pain, function, and quality of life. Am J Sports Med 2010;38:1456-1461.

27. Fedoriw WW, Ramkumar P, McCulloch PC, Lintner DM. Return to play after treatment of superior labral tears in professional baseball players. Am J Sports Med 2014;42: 1155-1160.

28. Bain GI, Galley IJ, Singh C, Carter C, Eng K. Anatomic study of the superior glenoid labrum. Clin Anat 2013;26: 367-376.

29. Cicchetti DV. Guidelines, criteria, and rules of thumb for evaluating normed and standardized assessment instruments in psychology. Psychol Assess 1994;6:284-290.

30. Sciascia AD, Myers N, Kibler WB, Uhl TL. Return to preinjury levels of play following superior labral repair in 
overhead athletes: A systematic review. J Ath Train 2015;50:767-777.

31. Hwang E, Hughes RE, Palmer ML, Carpenter JE. Effects of biceps tension on the torn superior glenoid labrum. J Orthop Res 2015:33:1545-1551.

32. Kibler WB. What is a clinically important superior labrum anterior to posterior tear?Pagano MW, Hart RA, eds. Instructional course lectures, Vol. 62 Rosemont, IL: American Academy of Orthopaedic Surgeons, 2013;483-489;Vol. 62, 2013.

33. Lippitt S, Vanderhooft JE, Harris SL, Sidles JA, Harryman DT II, Matsen FA III. Glenohumeral stability from concavity-compression: A quantitative analysis. J Shoulder Elbow Surg 1993;2:27-35.

34. Lippitt SB, Matsen FA. Mechanism of glenohumeral joint stability. Clin Orthop Rel Res 1993;291:20-28.

35. Maffet MW, Gartsman GM, Moseley B. Superior labrumbiceps tendon complex lesions of the shoulder. Am J Sports Med 1995;23:93-98.

36. Clavert P. Glenoid labrum pathology. Orthop Traumatol Surg Res 2015;101:S19-S24.

37. Bain GI, Phadnis J, Itoi E, et al. Shoulder crane: A concept of suspension, stability, control, and motion [published online April 8, 2019]. Joint Dis Orthop Sports Med. https:// doi.org/10.1136/jisakos-2017-000187.

38. Harryman DT II, Sidles JA, Clark JM, McQuade KJ, Gibb TD, Matsen FA Iii. Translation of the humeral head on the glenoid with passive glenohumeral motion. J Bone Joint Surg Am 1990;72:1334-1343.

39. Speer KP, Hannafin JA, Altchek DW, Warren RF. An evaluation of the shoulder relocation test. Am J Sports Med 1994;22:177-183.

40. Speer KP, Deng X, Borrero S, Torzilli PA, Altchek DA, Warren RF. Biomechanical evaluation of a simulated Bankart lesion. J Bone Joint Surg Am 1994;76: 1819-1826.

41. Burkhart SS, Morgan CD. The peel-back mechanism: Its role in producing and extending posterior type II SLAP lesions and its effect on SLAP repair rehabilitation. Arthroscopy 1998; 14:637-640.

42. Nishida K, Hashizume H, Toda K, Inoue H. Histologic and scanning electron microscopic study of the glenoid labrum. J Shoulder Elbow Surg 1996;5:132-138.

43. Burkhart SS, Morgan CD, Kibler WB. The disabled throwing shoulder: Spectrum of pathology Part I: Pathoanatomy and biomechanics. Arthroscopy 2003;19: 404-420.

44. Veeger HEJ, van der Helm FCT. Shoulder function: The perfect compromise between mobility and stability. J Biomech 2007;40:2119-2129.

45. Koga A, Itoigawa Y, Wada T, et al. Anatomic analysis of the attachment of the posteroinferior labrum and capsule to the glenoid: A cadaveric study. Arthroscopy 2020;36: 2814-2819. 